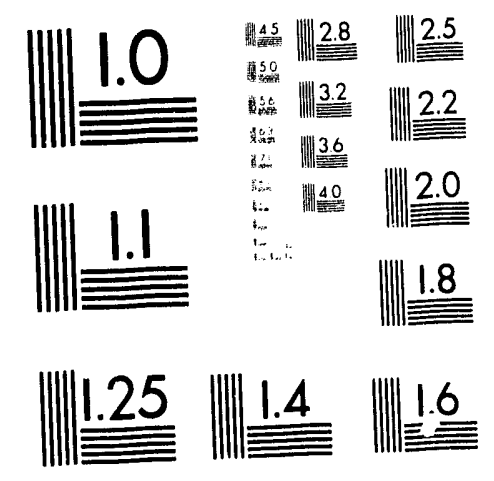



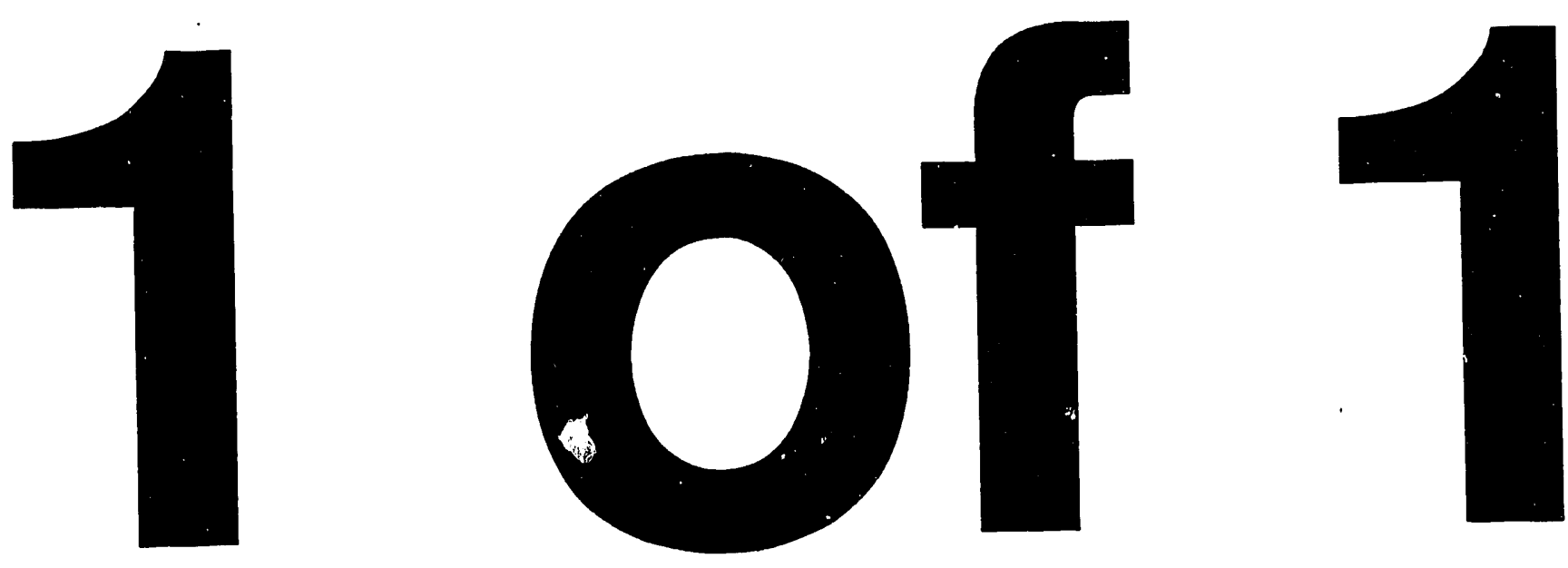
DOE / PC92544-4

\section{VAPOR PRESSURES AND HEATS OF VAPORIZATION OF PRIMARY COAL TARS}

E. M. SUUBERG (PRINCIPAL INVESTIGATOR)

V. OJA (GRADUATE STUDENT)

W.D. LILLY (STAFF)

DIVISION OF ENGINEERING

BROWN UNIVERSITY

PROVIDENCE, RI 02912

TEL. (401) 863-1420

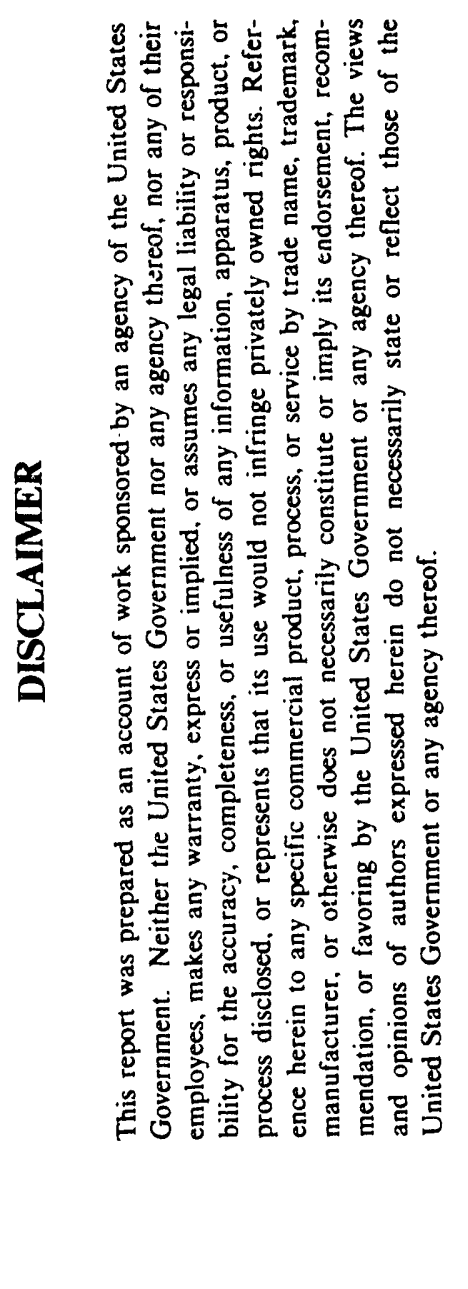

PREPARED FOR:

U. S. DEPT. OF ENERGY

PITTSBURGH ENERGY TECHNOLOGY CENTER

QUARTERLY TECHNICAL PROGRESS REPORT

1 APRIL, 1993- 30 JUNE, 1993

P.O. BOX 10940

PITTSBURGH, PA 15236

DR. KAMALENDU DAS, METC, MORGANTOWN, WV TECHNICAL PROJECT OFFICER

"US/DOE Patent Clearance is not required prior to the publication of this document"

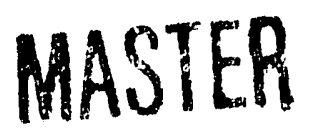




\section{General Background}

As the world continues to deplete its petroleum reserves, then heavy crude oil, coal liquids, and other heavy fossil fuels may be required to meet the world energy needs. Heavy fossil fuels contain molecules that are large and more aromatic and that contain more heteroatoms than those found in liquid crude oil.

There is also significant current interest in general area of coal pyrolysis, particularly with with respect to comprehensive models of this complicated phenomenon. This interest derives from central role of pyrolysis in all thermally driven coal conversion processes - gasification, combustion, liquefaction, mild gasification, or thermal benefication. There remain several key data needs in these application areas. Among them is a need for more reliable correlation for prediction of vapor pressure of heavy, primary coal tars. Such information is important in design of all coal conversion processes, in which the volatility of tarry products is of major concern.

The vapor pressure correlations that exist at present for coal tars are very crude and they are not considered reliable to even an order of magnitude when applied to tars. Sophisticated general correlative approaches are slowly being developed, based upon group contribution methods, or based upon some key functional features of the molecules. These are as yet difficult to apply to coal tars. The detailed group contribution methods, in which fairly precise structural information is needed, do not lend themselves well for application to very complex, poorly characterized coal tars. The methods based upon more global types of characterizations have not yet dealt much with the question of oxygenated functional groups. In short, only very limited correlations exist, and these are not considered reliable to even an order of magnitude when applied to tars.

The present project seeks to address this important gap in the near term by direct measurement of vapor pressures of coal tar fractions, by application of well-established techniques and modifications thereof. The principal objectives of the program are to: 1) obtain data on the vapor pressures and heats of vapcrization of tars from a range of ranks of coal, 2) develop correlations based on a minimum set of conveniently measurable characteristics of the t?rs, 3) develop equipment that would allow performing such measurements in a reliable, straightforward fashion. 


\section{Results from this Quarter}

A significant amount of time has been devoted during this quarter to preparing the equipment for production of the tars to be measured, and preparing the equipment for measurements by the so-called Knudsen effusion method. This method will be summarized below.

\subsection{The Knudsen method}

The various effusion methods are based on the effusion of the vaporized substance from a surface, or through an orifice [ 1]. One of these is the Knudsen method [ 2, 3], in which the substance effuses through small holes of known area. The principal applications of this method have been in the determination of the vapor pressure of metals such as mercury, cadmium, zinc and lead. It has however been equally effective for obtaining the vapor pressure of organic crystals and compounds $[4,5,6,7,8,9,20]$.

The Knudsen method is used for the measurement of low vapor pressures in the range from 1 to $10^{-6} \mathrm{~mm} \mathrm{Hg}$ under molecular flow conditions (pressures inside and outside cell are low enough for gas molecules make collisions only with the walls of the cell) and involves measurement of the rate of loss of molecules of the evaporating substance, leaving the opening of an effusion cell. Measurement are made under isothermal conditions, with weight loss being recorded as a function of time simultaneously with sample temperature, and with pressure measured outside the effusion cell.

The basic theory of the effusion method was reviewed in the literature $[2,3,12]$. The basic theory of method is based upon the kinetic theory of gases, from which Knudsen derived an expression for the slow isothermal flow out of a cell with a small hole in it. According to Knudsen [ 1] the vapor pressure of a material in the cell can be calculated from his result, using the equation :

$$
P_{1}-P_{2}=\frac{G}{t} \frac{w_{1}+w_{2}}{\sqrt{\rho}}
$$

$P_{1} \quad$ is the pressure of saturated vapor inside the cell

$\mathrm{P}_{2} \quad$ is the pressure outside of the effusion cell

$\mathrm{w}_{1}$ is the resistance of hole

$w_{2} \quad$ is the resistance of tube

$\mathrm{G}$ is the mass lost by effusion

$t \quad$ is the effusion time

$p \quad$ is the density of the vapor at the temperature of experiment and at the 
pressure 1 dyne per sq. $\mathrm{cm}$.

The relation simplifies upon applying the ideal gas law and neglecting $P_{2}$ (i.e. $P_{1} \gg$ $\left.\mathrm{P}_{2}\right)$ :

$$
P=\frac{m}{t A_{0}}\left(\frac{2 \pi R T}{M}\right)^{1 / 2}
$$

, which is called the ideal Knudsen equation, and in which the variables have the following definitions:

$\begin{array}{ll}P & \text { vapor pressure [ atm ] } \\ m & \text { weight loss during the effusion time interval [ } \mathrm{g}] \\ \mathrm{A}_{0} & \text { orifice area }\left[\mathrm{cm}^{2}\right] \\ M & \text { molecular weight }[\mathrm{g} / \mathrm{mol}] \\ \mathrm{t} & \text { effusion time }[\mathrm{sec}] \\ T & \text { absolute temperature }[\mathrm{K}]\end{array}$

It is assumed when applying this equation to the effusion process:

-That the equilibrium vapor pressure of the effusing species obtains within the cell.

- That the orifice walls do not intercept and return into the cell an appreciable fraction of molecular current entering the hole. This equation is strictly applicable only if the thickness of the wall, in which the orifice of area $\pi \mathrm{a}^{2}$ is located, is vanishingly small compared to the hole's radius a. It mean that the orifice should locate in infinitely thin wall or be made so that the edge is exceedingly sharp ( knife edge orifice).

-That there is no back flux into the orifice exit - no molecules returned once they have passed through the orifice

-That the pressure outside the cell is much lower than the pressure of saturated vapor inside the cell; i.e. $\mathrm{P}_{1} \gg \mathrm{P}_{2}$.

- That the number of intermolecular collisions in the vapor phase occurring within the orifice is neglible, which will be true if the molecular mean free path in the vapor is long compared to the the orifice diameter. Knudsen's [1] criterion is adopted that molecular flow ( collision free ) occurs when $\lambda \geq D$, where $\lambda$ is the mean free path of vapor molecules within the cell and $\mathrm{D}$ is the diameter of the orifice. 


\subsection{Complications Inherent in the effusion method}

\subsubsection{Nonideal orifice, Clausing factor, effect of orifice geometry}

In the particular case considered here, the orifice is not located in an infinitely thin wall and it has the form of channel of finite length. Some molecules which enter the orifice will not escape, but will strike the wall of the orifice and be reflected diffusely from it. Clausing rewrote the Knudsen equation to include a transmission probability $\mathrm{W}_{0}$, now commonly called the Clausing factor:

$$
\mathrm{P}=\frac{\mathrm{m}}{\mathrm{t} \mathrm{W}_{0} \mathrm{~A}_{0}}\left(\frac{2 \pi \mathrm{RT}}{\mathrm{M}}\right)^{1 / 2}
$$

Clausing assumed that molecules enter the orifice from an isotropic gas phase from a random direction, according to the cosine law. For a non-ideal orifice this requires a decrease in the net rate of effusion through a hole of finite length.

Thus the term $\mathrm{W}_{0}$ has values in the range zero to unity and it is introduced to correct the fact that the number of molecules effusing may be less for a cell whose orifice does not have infinitely thin walls. The term may be physically understood as a probability that a molecule entering the orifice from the effusion chamber will reach the exterior of the orifice. The Clausing factor has been shown to be a function of the ratio of the length to the radius of a cylindrical cell through which the molecules travel. Values of $W_{0}$ for cylindrical and rectangular orifices are given and described by Clausing [13], Dushman [1], Kennard [14]. Typical values [ 1 ] are shown in Table 1.

Table 1.

Values of Clausing's Factor $W_{0}$ for a Series of Values of $(L / a)$

\begin{tabular}{llll}
\hline L/a & \multicolumn{1}{c}{$\mathrm{W}_{0}$} & $\mathrm{~L} / \mathrm{a}$ & $\mathrm{W}_{0}$ \\
\hline 0 & 1 & 0.6 & 0.7711 \\
0.1 & 0.9524 & 0.7 & 0.7434 \\
0.2 & 0.9092 & 0.8 & 0.7177 \\
0.3 & 0.8699 & 0.9 & 0.6940 \\
0.4 & 0.8341 & 1.0 & 0.6720 \\
0.5 & 0.8013 & 2.0 & 0.5136 \\
\hline
\end{tabular}

where $\mathrm{L}$ is the length of the finite tube or the thickness of the plate, where the hole is drilled and $\mathrm{a}$ is the diameter of the hole. 


\subsubsection{Evaporation coefficient}

In the usual experiment, a cell is provided at one end with a small opening through which the effusion process occurs into vacuum of $10^{-6} \mathrm{~mm} \mathrm{Hg}$ or less outside the cell. If there were not an opening in the cell, any pure material would come to equilibrium with the rates of vaporization and condensation equivalent at the pressure $\mathrm{P}_{\mathrm{eq}}$. The material balance can be written:

$$
\text { rate of vaporization }=\text { rate of condensation }
$$

Once an opening has been provided for molecules to effuse, there is no longer a closed system and the pressure over the sample is not $\mathrm{P}_{\mathrm{eq}}$ but something less than this, a steady state pressure $\mathrm{P}_{\text {ss. }}$.

In a vaporization process which has attained equilibrium, the number of molecules which strike the surface of the condensed phase and remain must equal the number of molecules which leave the surface. All of the molecules which strike the surface do not, however, stick so that there is less than unity probability of condensation for every incoming molecule. The same is true under steady state, as opposed to true equilibrium conditions. It is the steady state condition that is of interest in an effusion-type experiment, in which vapor molecules are lost from the effusion hole. The reasons for less than $100 \%$ sticking probability (condensation) may be structural. Some gas molecules may have to have a specific orientation or vibration amplitude in their approach to the condensed phase. Other possibilities include the fact that some molecules may have a different dimensions in the vapor phase from those in the condensed phase, that they may dimerize, or dissociate to completely different types of molecules structurally and find it difficult to fit into the surface phase.

According to kinetic theory, equilibrium is still described as a balance between the rate of evaporation and the rate of condensation. One may write the material balance for the effusion cell:

rate of vaporization - rate of condensation $=$ rate of effusion

The rate of effusion through the orifice area is the difference between the rate of evaporation and condensation. This is clearly a different situation than presented above for true equilibrium, and only approaches that ideal when the rate of effusion is quite small compared to the rate of escape of molecules from the surface by evaporation and small compared to the rate of condensation onto the surface. 
Langmuir [15] has shown that for metal atoms condensing on a surface the value of condensation coefficient (sticking probability) $\alpha$ may be assumed equal to 1 and Verhoek and Marshal [16] showed that the same assumption is justified in the high boiling point organic liquids. Accurate values of condensation coefficients are generally not available. Plausible reasons for $\alpha$ to be close to unity have been advanced, but for the majority of cases it is probably between 0.7 to 1 ; for vaporization of species whose molecular structure is quite different in the gas than in solid or liquid (e.g. dimer liquids), $\alpha$ may be very small, $0.001<\alpha<0.1$.

When this factor is considered, equations for determination of a $\mathrm{P}_{\mathrm{eq}}$ must explicitly include consideration of the dynamic equilibrium at the surface of the vaporizing liquid. This requires inclusion of a term that recognizes that the surface evaporation and condensation processes have a rate proportional to the surface area of the liquid, $A_{\mathbf{S}}$. The degree to which true equilibrium is maintained also depends upon the sticking probability being high enough so that effusion does not compete as a vapor phase loss process. Thus the corrected equation has the overall form:

$$
P_{e q}=P_{e q}\left(P_{S s}, \alpha, W_{0}, A_{0}, A_{S}\right)
$$

where $A_{S}$ is the sample area from which evaporation occurs, but usually it is taken as the cross sectional area of the cell (in case of cylindrical cells ). The cell geometry also affects the calculation.

In our case, we will be operating with organic liquids in which $\alpha$ can be assumed close to unity, and we will also be operating in a regime for which the rate of vaporization from the surface can be assumed much greater than the rate of effusion (i.e. that true equilibrium can be maintained). Thus $P_{\text {eq }}$ can be well approximated by $P_{S S}$ [12].

\subsubsection{Effect of cell geometry}

The geometry of cell body and the location and area of the sample within it affect the total rate of flow and several authors have introduced the transmission factor $\mathrm{W}_{\mathrm{c}}$ or Clausing factor for a cell, which is the probability that a molecule leaving the bottom of the cell will reach the orifice. In this case the calculation of $\mathrm{P}_{\mathrm{eq}}$ involves a relationship of form:

$$
P_{\text {eq }}=P_{e q}\left(P_{s s}, W_{0}, W_{c}, A_{0}, A_{s}\right)
$$

The equations have been developed for a cylindrical cell by Clausing [13], Mortzefeld [17], Whitman [18], Balson [11]. Carlson [21] has presented a mathematically rigorous 
derivation of the distribution of the gas within spherical and cylindrical cells.

In addition to the above processes, there may exist some others that can cloud interpretation of results, for example interaction between walls and effusing molecules, surface diffusion, specular reflection, reaction at the walls. We do not believe that these will be significant in our case.

\subsection{Conclusion}

It has been concluded that the effusion method is a quite accurate technique for measuring vapor pressures of pure component in the range from 1 to $10^{-6} \mathrm{~mm} \mathrm{Hg}$, but the technique has some complications:

Technical considerations:

-A high vacuum system must be used, and the vacuum outside the cell ( $10^{-6}$ to $10^{-7}$ $\mathrm{mm} \mathrm{Hg}$ ) should be achieved quickly, so that the beginning of an experiment can be registered with sufficient temporal accuracy. It is recommended that pressure external to the cell be at least one order of magnitude below the vapor pressure to be determined [10].

-Maintaining temperature with a high accuracy is difficult. It is noted by Wiedemann [6] that the change of a few tenths $\mathrm{K}^{\circ}$ will alter the vaporization rate and lead to Significantly erroneous values of vapor pressure. The sensitivity to temperature error will be calculated in our studies.

-A highly sensitive microbalance is required; typical values taken from the literature show how the sensitivity of the balance affects the lower limit of vapor pressure that can be examined :

$$
\begin{array}{cc}
\text { Effusion rate }=\quad 10^{-9} \mathrm{~g} / \mathrm{sec} \text { allows measuring a pressure of } & 10^{-6} \mathrm{mmHg} \\
10-8 \mathrm{~g} / \mathrm{sec} & 10^{-5} \mathrm{mmHg} \\
10-7 \mathrm{~g} / \mathrm{sec} & 10^{-4} \mathrm{~mm} \mathrm{Hg}
\end{array}
$$

Our microbalance can reliably record changes of fractions of a micrograms, so in an experiment of one hour duration, we should be capable of operation at the lowest pressure levels shown above.

- A cold trap near the orifice of the Knudsen cell is recommend to condense the vaporized compound and assist in the external pressure low. We have not made provision for a cold trap near the cell, but we will rely upon high pumping rates and a condenser slightly downstream of the cell to give us the necessarily low pressures outside of the cell.

- Orifice diameter selection - Morecroft has showed that the vapor pressure measurement is independent of hole size [5]. It is has been suggested that it is necessary that the mean free path of the molecules is larger than orifice diameter in order employ the Knudsen 
formula (this would normally be interpreted as mean free path ten times large than hole diameter ). Good result can, however, still be obtained when the diameter is about same as the mean free path [5]. On the other hand, since the effusion rate is very small at low pressures, a large orifice (diameter 3 to $5 \mathrm{~mm}$ ) is required to give reasonable rates [6], but very large holes can yield considerably lower values for vapor pressures, owing to selfcooling of the sample and failure to maintain equilibrium [10]. In addition to slow effusion rates, other problems also exist with small orifices. For example, the existence of small leaks in the cell can cause problems if the effusion hole has a small size, since the calculated vapor pressure will be considerably higher than the true value, as a result of using the "wrong" orifice area. It is reported by Winterbottom and Hirt [19] that the surface diffusion increases as the radius of the hole decreases.

The determination of the Clausing probability factor $\mathrm{W}_{0}$ for the orifice is one of important requirements for this measurement. There are two approaches. Either the cell can be made so that the plate thickness and the hole diameter are accurately known and $\mathrm{W}_{0}$ can be calculated using known expressions, or the effusion cell can be calibrated using reference materials for which pressures are accurately known and $W_{0}$ is calculated using equation 3:

$$
P=\frac{m}{t W_{0} A_{0}}\left(\frac{2 \pi R T}{M}\right)^{1 / 2}
$$

Equation 3 has generally been used to calculate equilibrium vapor pressures of organic compounds [5], [6], [7], [8], [9], [20], but as was noted elsewhere this steady state pressure $P_{S s}$ may be expected to be slightly less than equilibrium pressure $P e q$. On the other hand, accurate values for $\alpha$ and Peq for making further corrections cannot normally be found [10].

\section{Qur Approach}

It is thus concluded that equation 3 should be used for our proposes, since the corrections described in 2.2.2 and 2.2.3 are difficult to perform, and they are probably not very large in any case. How much error they may cause can be empirically evaluated by working at different effusion rates and with different hole/cell geometries.

The effusion method as implemented in recent years generally involves use of a standard thermogravimetric analyzer (TGA). We have two at our disposal for this work, one is 
based upon a Cahn 2000 recording microbalance, and the other is a DuPont 951 system. System parameters are shown below for both:

\section{CAHN 2000 Microbalance}

\begin{tabular}{ll}
\hline Sensitivity & $0.1 \mu \mathrm{g}$ \\
Capacity & $1.5 \mathrm{~g}$ \\
vacuum & $10-7 \mathrm{~mm} \mathrm{Hg}$ \\
\hline
\end{tabular}

\section{Du Pont 951 Thermogravimetric Analyser}

\begin{tabular}{ll}
\hline Sensitivity & $5 \mu \mathrm{g}$ \\
Capacity & $0.5 \mathrm{~g}$ \\
vacuum & $1 \mathrm{~mm} \mathrm{Hg}$ \\
\hline
\end{tabular}

It can see that Cahn microbalance is more suitable for our proposes, it has higher sensitivity and capacity, and its vacuum envelope (as modified) will be the only suitable choice.

The general schematic of the effusion apparatus is shown in Figure 1.

\subsection{Result to Date}

Most of results of present quarter have been concerned with the development of experimental methods and modification and rebuilding microbalance, saturation, calibration and rapid pyrolysis devices.

\subsubsection{Continuous flow saturation method}

This method was described in the previous quarterly report. Based on work done in this quarter:

a) It was decided to use and put in order a Perkin-Elmer Sigma 3 Gas Chromatograph instead of an older Model 3920 which exhibits unacceptable noise of $0.1 \mathrm{mV}$, (comparable to signals to be measured) at high temperatures. This is necessary to obtain reliable values at low vapor pressures.

b) The calibration mixtures for this technique will be provided by KIN-TEK Precision 
Calibration Systems, which provide reliable constant flows in the 0.1 to $1 \mathrm{ppm}$ range. The calibration material will be toluene.

\subsubsection{Effusion method}

We have had difficulties in rebuilding the existing TGA system and pumping system to the necessary high vacuum specifications. There is no fundamental problem with getting these in working order, rather, it is a question of further funds and time expenditure.

\subsubsection{Sample Preparation system}

The heated wire mesh pyrolysis system has been substantially rebuilt, to permit more efficient collection of the quantities of the tar necessary for the vapor pressure tests.

\section{Plans for the Neyt Quarter}

During the next quarter we plan to continue to rebuild and modify the Cahn 2000 recording microbalance, saturation, calibration and rapid pyrolysis devices and continue develop the effusion and saturation methods.

\section{Literature}

1. Dushman, S.," Scientific foundations of vacuun technique," New - York, John Wiley \& Sons, 2nd edition, 1962

2. Knudsen, M., Ann. Physik., 28, 999, 1909.

3. Knudsen, M., Ann. Physik., 29, 179, 1909.

4. Swan, T.H., Mack E., J. Am. Chem. Soc., 47, 2112,1925.

5. Morecroft, D.W., J. Chem. Eng., 9, 488, 1964.

6. Wiedemann H.G., Thermochim. Acta, 3, 355, 1972.

7. DePablo, R. S., J. Chem. Eng., 21, 141, 1976.

8. DeKruif, C.G., J. Chem. Thermodynamics, 12, 243, 1980.

9. Colomina, M., Jimenez, P., Perez-Ossorio, R. and M.V.Roux, J. Chem. Thermodynamics, 20, 575, 1980.

10. Ribero Da Silva, M. and Monte, M., Thermochim. Acta, 171, 169, 1990.

11. Balson, E.W., J. Phys. Chem., 65, 115, 1961.

12. Hollahan, J., J. Chem. Educ., 39, 23, 1962. 
13. Clausing, P., Ann. Physik., 12, 961, 1932.

14. Kennard, E.H.", Kinetic Theory of Gases," McGraw-Hill, New-York, 1938.

15. Langmuir, I., Phys. Rev., 2, 329, 1913.

16. Verhoek, F.H. and Marshall, A.L., J. Am. Chem. Soc., 61, 2737,1939.

17. Motzfeldt, K., J. Phys. Chem., 59, 139, 1955.

18. Whitman, C.J., J. Chem. Phys., 20, 161, 1952.

19. Winterbottom, W. and Hirt, J., J. Chem. Phys., 37, 784, 1962.

20. Colomina, M., Jimenez, P. and Turrion, C., J. Chem. Thermodynamics, 14, 779, 1982.

21. Carlson, K. D., Gilles, P. W. and Thorn, K.J., J. Chem. Phys., 38, 2064, 1963. 


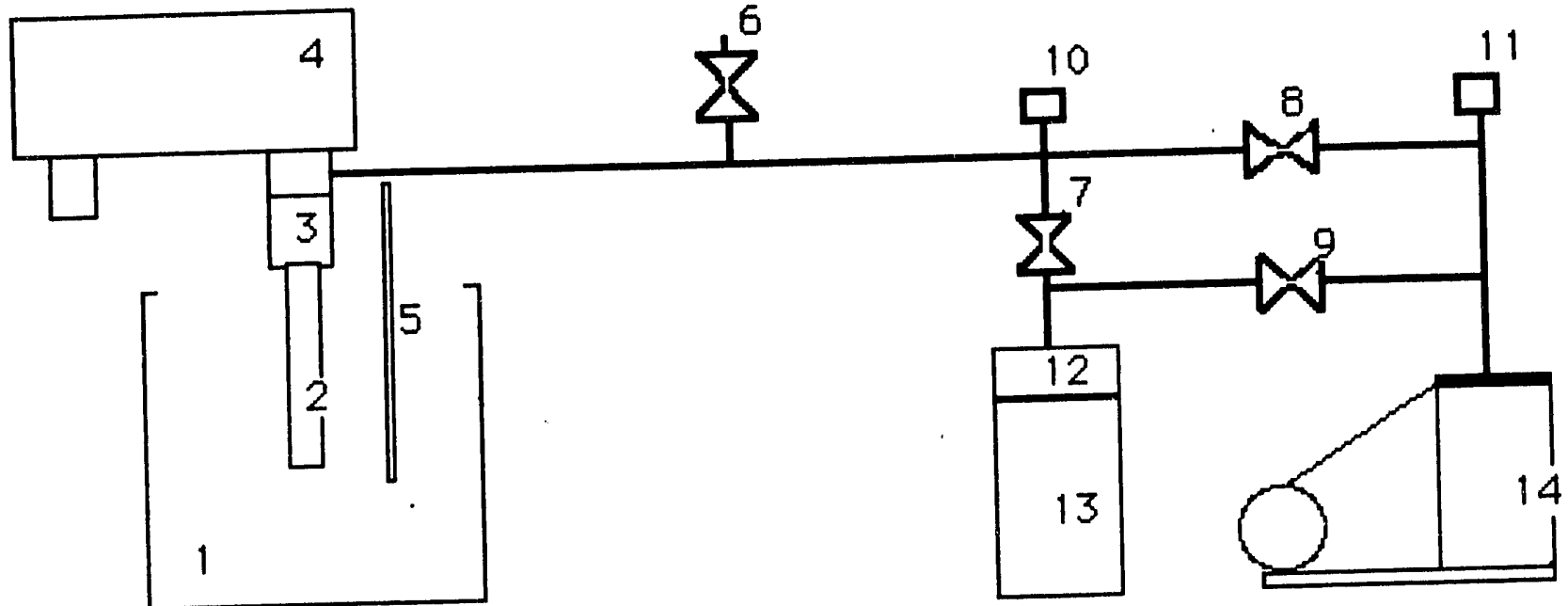

1 Thermostatic bath

2 glass tube

3 cold trap

4 TGA

5 mercury thermometer
$6,7,8,9$

10,11

12

13

14 valves vacuum gouges liquid nitrogen trap oil diffusion pump mechanical pump

Fig. 1. General scheme of the effusion apparatus 

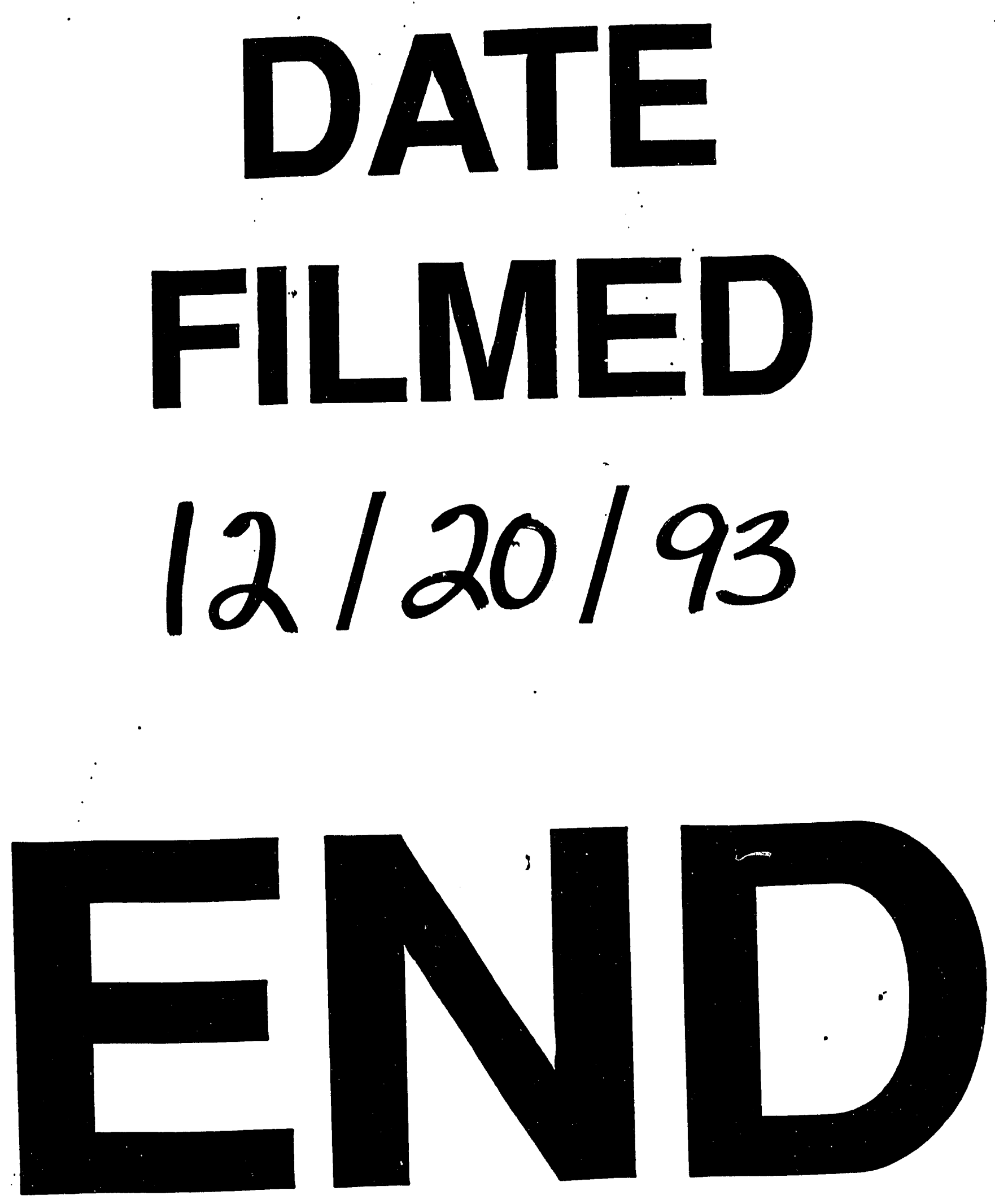
